\title{
Test de Detección para el diagnóstico positivo de la ruptura prematura de las membranas ovulares ${ }^{(*)}$
}

\author{
Doctor Edgard Cobo C., M. D.
}

Residente de la Clínica de Maternidad de Cali.-Instructor del Departamen. to de Obstetricia y Ginecología de la Escuela de Medicina de la Universidad del Valle.

La ruptura prematura de membranas en sí misma tiene esca_ sa significación. Sin embargo, los problemas que pueden resultar como complicaciones de ella, pueden alcanzar tales proporciones que su profilaxia se convierte en una obligación absoluta del es. pecialista. De esto se desprende la considerable importancia que dentro de la práctica obstétrica moderna ha adquirido la determinación rápida y exacta del estado real de las membranas ovu. lares en las últimas semanas del embarazo, ya que su ruptura prematura constituye en un apreciable número de casos una com. plicación de repercusiones severas sobre pronóstico del parto.

En estudios recientes se ha demostrado que la incidencia de esta entidad corresponde aproximadamente a un $10 \%$ de los ca. sos; asignándosele un $60 \%$ de este último dato al tipo de la rup. tura prematura, es decir, aquella que se presenta antes de la ini_ ciación del trabajo de parto; y un $40 \%$ al tipo de la ruptura precoz, o sea ia que se presenta una vez iniciado éste. De una de_ terminación positiva de estos tipos de ruptura de las membranas ovulares surge, pues, la prevención de todos los problemas obstétricos inherentes a ella, durante el período latente, el trabajo de parto y el parto mismo, y que van en orden de frecuencia desde el edema de cuello y los prolapsos del cordón y de las extremi. cades del feto, hasta los trastornos profundos de los mecanis. mos fisiológicos de la respiración y de la coagulación sanguínea, como son el embolismo amniótico y la afibrinogenemia.

* Trabajo leído en la Sociedad de Obstetricia y Ginecología del Valle y enviado a la Revista para su publicación. 
Desde 1936 Numers describió un sistema de laboratorio ten. diente a la comprobación exacta de la ruptura prematura de membranas, consistente en la elaboración de un frotis vaginal te. rído con una solución de Sudán III, con el objeto de evidenciar la presencia de glóbulos grasos provenientes del vermix gaseoso que recubre la piel del feto durante las últimas semanas del em. barazo. Dos años más tarde, en 1938. Baptisti presentó un siste. ma más sencillo, que consistía en la determinación del pH vagi_ nal con tiras de papel indicador, fundamentado en el hecho real del cambio del medio vaginal, de ácido alcalino, una vez estable. cido el drenaje de líquido amniótico a través de la vagina. Más recientemente, en 1955, Goldfine reporta una nueva técnica apli_ cando al frotis vaginal hecho en las últimas semanas del emba. 1azo, el sistema de tinción de Papanicolau. El diagnóstico positi. vo se estructura en este sistema, sobre la desaparición a medi. da que avanza el drenaje amniótico, de las células vaginales su_ perficiales y de los leucocitos, al tiempo que van apareciendo en menor cuantía células pertenecientes a los estratos interme. dios de la vagina. Estos cambios son producidos por el barrido mecánico producido por la salida del líquido amniótico y produ_ cen, cuando ya han trancurrido algunas horas después de la rup_ tura de las membranas, lo que Goldfine ha llamado un "frotis limpio", en el que no se observan sino algunas células interme. dias y muy pocos leucocitos. En algunos casos es posible eviden. ciar por este sistema células de descamación del epitelio fetal, y en ocasiones lanugo.

\section{MATERIAL Y METODO}

Para llevar a cabo un plan de trabajo dirigido a la aplica. ción en nuestro medio de estos sistemas de laboratorio usados universalmente en el problema diagnóstico de la ruptura pre_ matura de membranas, se estableció un procedimiento consisten. te en la asociación de los tres métodos descritos atrás, aprove. chando la sencillez de los dos primeros y asimilando a ellos el criterio histológico del tercero, que como se vio en su descripción, exige la presencia de personal entrenado en citología vaginal y además requiere dos horas o más para su elaboración cuidadosa. Previo a la iniciación del test de detección, se hizo un frotis va_ ginal en fresco, diluyendo el contenido del fondo de saco poste. rior en solución salina fisiológica.

Se escogieron entonces al azar treinta pacientes admitidas $\epsilon n$ nuestro servicio de obstetricia durante el año de 1957, con 
diagnóstico clínico de ruptura prematura de membranas y se so. metieron al sistema del Test de Detección de acuerdo con la si. guiente técnica:

1a Colocación de la paciente en posición ginecológica, ha. ciendo lavado de genitales externos con agua estéril, sin usar ningún antiséptico local, y cubriéndolos arriba y abajo con cam. pos estériles.

2a Aplicación de un espéculo vaginal seco, hasta visualizar el cuello uterino y el fondo de saco posterior.

$3^{\text {a }}$ Introducción de una pinza de curación armada de una pequeña tira de papel indicador, humedeciéndola con el líquido en. contrado en el cuello o en el fondo de saco posterior.

4 a Lectura en la escala colorimétrica del resultado obtenido en el papel un minuto después de su impregnación.

$5^{\text {a }}$ Introducción de una pipeta.gotero hasta el fondo de saco posterior y aspiración de su contenido.

$6^{\text {a }}$ Elaboración de un frotis vaginal en fresco, dejando caer 2 gotas de contenido vaginal sobre una lámina y disolviéndolas en 2 gotas de solución salina fisiológica, antis de aplicar sobre fllas la laminilla.

7ạ Elaboración del frotis vaginal por coloración. extendien. do el resto del material aspirado sobre una lámina con el tallo de la pipeta_gotero, llevándolo de extramo a extremo de la lámi. na. Se seca el extendido al aire o al mechoro.

8a Aplicación sobre la lámina de una solución de 0.3 gramos de Sudán III en 100 c. c. de alcohol al $70 \%$. Esta solución se deja sobre la lámina 4 minutos.

9a Lectura del frotis.

La lectura de los frotis se hizo según la técnica de rutina pa_ ra el frotis en fresco y de la misma manera, usando pequeño y gran aumento, para el frotis teñido con Sudán III. Con la obser. vación del primer frotis se busca descartar la presencia de trichomonas vaginalis, demasiado frecuentes intre nosotros y que constituyen una de las entidadies con las que debe hacerse el diagnóstico diferencial con la ruptura prematura de membranas. La observación del frotis teñido con Sudán III se hizo sobre la búsqueda, en primer término, de glóbulos grasos aue aparecen como pequeñas gotas salientes, coloreadas de rojo anaranjado, de límites netos y forma globulosa. En segundo término se pro. cedió a la determinación de células del epitelio vaginal, sin es. tablecer diferenciación por estratos, ya que no puede hacerse por esta coloración; y a la observación de los leucocitos. De todos 
estos elementos se hizo una determinación cuantitativa aproxi_ mada, de acuerdo con el siguiente esquema:

Glóbulos grasos: (+) Para los frotis con un glóbulo graso menos por campo, sobre un total de 10 campos examinados.

$\left({ }^{+}\right)$Para 2 glóbulos grasos por campo.

$(+++)$ Para 3 glóbulos grasos por campo.

$(++++)$ Para 4 glóbulos grasos o más por campo.

Células epiteliales: (+) Cuando la placa obtenida correspon. de a lo que Goldfine ha denominado un "frotis limpio", es decir, cuando el barrido mecánico ha hecho desaparecer prácticamen. te estos elementos de la cavidad vaginal.

$\left({ }^{+}+\right)$Cuando el número de células no pasa de 10 por campo.

$(+++)$ Cuando su número no pasa de 20 por campo.

$(++++)$ Cuando el número de elementos celulares es mayor de 20 por campo. Este tipo de frotis generalmente va asociado a

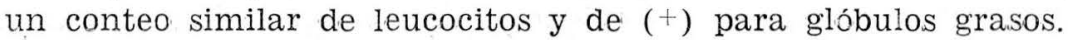

Leucocitos: $\left(^{+}\right)$El mismo criterio anterior.

La evaluación del período latente, es decir, del espacio trans. currido entre el momento de la ruptura y la iniciación del traba. jo de parto, se adjuntó siempre al diagnóstico, teniendo en cuen. ta la hora en que se hizo el frotis. Se obtuvo este dato combinan. do los hallazgos microscópicos con los datos suministrados por la anamnesis de la enferma. El resultado definitivo de la prue. ba se obtuvo siempre en un lapso de tiempo no mayor de $15 \mathrm{mi}$. nutos. Bajo el título de Test de Detección se reportaron a la his. toria clínica los resultados, de acuerdo el siguiente esquema:

\section{PRUEBA DE DETECCION}

pH. vaginal

FROTIS.

En fresco: Trichomonas vaginalis

Sudán III: Células epiteliales

Leucocitos

Glóbulos grasos

DIAGNOSTICO

OBSERVACIONES

\section{RESULTADOS}

Los 30 casos sometidos a este estudio fueron controlados rigu. rosamente durante el periodo latente, la iniciación del trabajo del parto, la evolución de éste y su culminación posterior. En general se obtuvieron partos espontáneos. Se practicó una operación ce. 
sárea con indicación principal independientie de la ruptura pre. matura de membranas y una gran extracción podálica por prolap_ so del cordón en un caso de ruptura precoz. El resto de los casos fue resuelto por aplicación de fórceps bajos y profilácticos, según la usanza más frecuente en nuestro medio. No se presenta un es_ tudio estadístico de los datos obtenidos, ya que la escasez de su número no permite un análisis demostrativo y porque, además, no es eso realmente el objetivo de este trabajo. En todos los casos, y a pesar de no encontrar glóbulos grasos en el extendido vaginal, se hizo un diagnóstico positivo de ruptura prematura de membra. nas, basado en los demás elementos de juicio que esta asociación de sistemas puede proporcionar. En otros 2 casos se obtuvo, por medio del primer frotis, el diagnóstico de trichomoniasis vaginal, evidenciado mediante el hallazgo de los parásitos y ante la ausen. cia, en el segudo frotis, de los elementos característicos de la rup_ tura prematura de membranas.

He seleccionado 4 de los frotis obtenidos por la coloración de Sudán III, tal vez los más demostrativos, y en los que puede apre. ciarse muy claramente el criterio histológico asimilado a este sis. tema, presentado a través de la evolución de un cuadro de ruptu_ ra de membranas ovulares acaecida prematuramente, seguido por medio de estas reproducciones fotográficas que corresponden, en su orden, a los siguientes diagnósticos:

CASO A.-HISTORIA NUMERO 176157.

\section{"TEST" DE DETECCION}

$\mathrm{pH}$ vaginal $\ldots \begin{array}{llll}\ldots & \ldots & 6.0\end{array}$

\section{FROTIS}

En fresco:

Trichomonas vagina-

lis ++++

Sudán III:

Células epiteliales ...t+++

Leucocitos ........ ${ }^{++++}$

Glóbulos grasos .......(一)

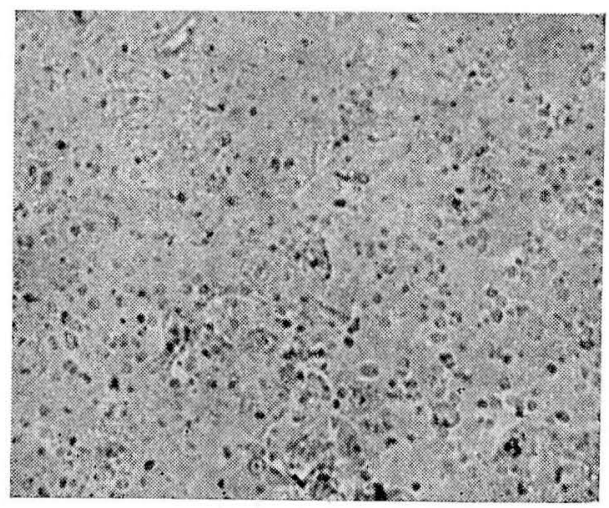

Diagnóstico: a) Membranas integras. b) Trichomoniasis va_ ginal. 
CASO B.-HISTORIA NUMERO 11661156

"TEST" DE DETECCION

pH vagina! ........ 8.0

FROTIS.

En fresco:

Trichomonas vagina_

lis $\ldots . . . . \ldots \ldots(-)$

Sudán III:

Células epiteliales.....$^{++}$

Leucocitos ........ ++++

Glóbulos grasos ......+

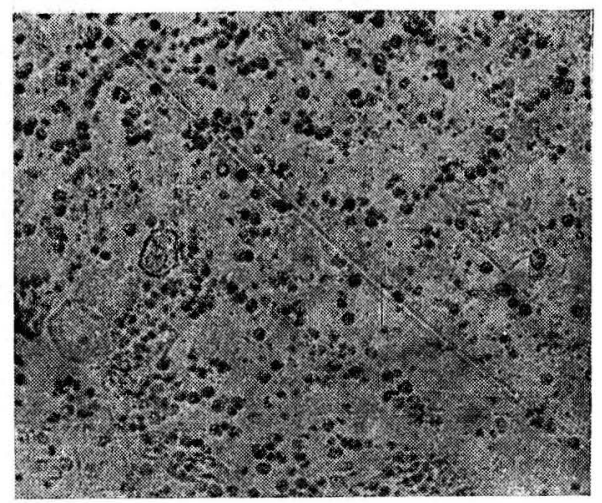

Diagnóstico: Ruptura prematura da membranas. Fase inicial.

CASO C.-HISTORIA NUMERO 20357

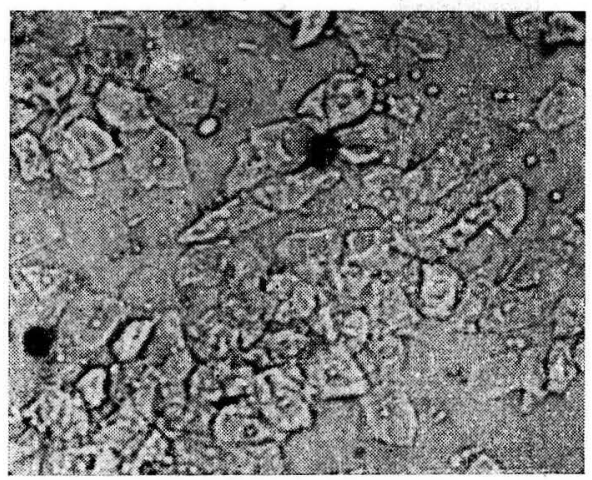

“TEST" DE DETECCION

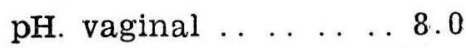

FROTIS.

En fresco:

Trichomonas vagina_

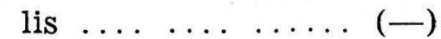

Sudán III:

Células epiteliales .....+++

Leucocitos ...........+ ++

Glóbulos grasos ....... .

Diagnóstico: Ruptura prematura de membranas. Período latente aproximado de 12 horas. Fase media. 
CASO D.-HISTORIA NUMERO 137357

"TEST" DE DETECCION.

$\mathrm{pH}$. vaginal $\ldots \ldots \ldots .8 .0$

\section{FROTIS}

En fresco:

Trichomonas vagina.

lis $\ldots . \ldots . \ldots .(-)$

Sudán III:

Células epiteliales ......+

Leucocitos .... ...... +

Glóbulos grasos ......++

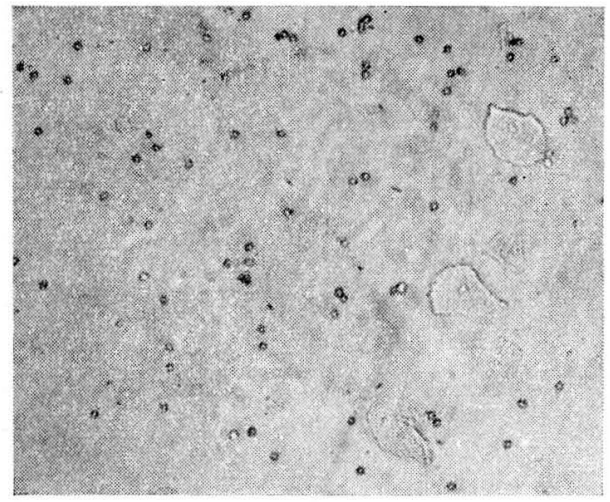

Diagnóstico: Ruptura prematura de membranas. Período la. tente aproximado de 36 horas. Fase tardía.

\section{COMENTARIO}

Como hemos visto, los datos clínicos que pueden llevar al diagnóstico de ruptura prematura de membranas son a menudo in. ciertos; al tiempo que la determinación rápida y exacta del es. tado real de ellas adquiere, desde el punto de vista preventivo, un valor cada vez mayor dentro de la práctica obstétrica moderna. Estas consideraciones imponen claramente la adopción de un sis. tema de diagnóstico positivo, eficaz y seguro, que lleve a la con_ firmación exacta de un caso de diagnóstico oscuro. Después de una revisión exhaustiva sobre el estado actual de los procedimien. tos diagnósticos certeros ante esta complicación obstétrica, y considerando a la vez las posibilidades que pueden existir en nuestro medio para su aplicación práctica, he creído encontrar un méto_ co ideal en la combinación de sistemas descrita atrás.

La determinación del $\mathrm{pH}$ vaginal obtenida por medio de sustancias indicadoras, da un promedio de positividad de $98 \%$, según su autor; al paso que la determinación de los glóbulos grasos pre. sentes en el extendido vaginal asegura un promedio de $99.3 \%$, una vez que el embarazo haya sobrepasado el límite de la 36a semana. Antes de este límite su valor es dudoso, dada la escasa cantidad de vermix caseoso que recubre la piel del feto. Por lo tanto, si se 
tienen en cuenta estos factores, el promedio de positividad aumen. ta considerablemente si se reúnen estos sistemas, ya que las cau. sas de error inherentes al primero pueden desaparecer ante la evi. dencia microscópica del segundo, y viceversa, la sospecha ante un extendido vaginal de interpretación dudosa, puede definirse me. diante la medición cuidadosa del pH vaginal.

Sin embargo, donde el sistema que propongo adquiere un va_ lor irrefutable, radica en su asimilación al sistema de Goldfine, con la certeza absoluta de un criterio histológico definido. Ya he mos visto que la elaboración del frotis vaginal para tinción con Papanicolau requiere por lo menos 2 horas para su resultado defi. nitivo, además de exigir para su manejo la presencia de personal entrenado suficientemente en citología vaginal. El "Test" de De. tección elimina estos inconvenientes, ya que no hace discrimina ción de los estratos de la mucosa vaginal y por lo tanto puede ser lé́do por quien lo haya visto pocas veces; solo requiere de 6 a 15 minutos para su interpretación global; tiene vigencia a cual_ quier edad del embarazo y por último hace, o por 10 menos lo ha hecho hasta ahora, una determinación absoluta del estado de las membranas ovulares durante la segunda mitad del embarazo.

De otra parte, como se vió en la descripción del método em. pleado en este trabajo, la ejecución simultánea de un frotis vagi. nal en fresco puede hacer un diagnóstico diferencial exacto con la trichomoniasis vaginal, demasiado frecuente entre nuestras pa. cientes.

\section{SUMARIO}

10 Se hace énfasis en la importancia del diagnóstico positivo de la ruptura prematura de membranas durante la segunda mitad del embarazo y se recalca la importancia que ella puede tener dentro de la práctica obstétrica moderna, orientada a la medicina preventiva.

2 을 Se presenta una asociación original de sistemas adoptados universalmente y se asimila a ellos un criterio histológico indepen_ diente del sistema de tinción de Papanicolau.

3 ํ. No se presentan análisis estadísticos, ya que el escaso nú_ mero de pacientes estudiadas en este trabajo no permite la elabo. ración de un estudio demostrativo y se deja este capítulo para ser presentado en comunicaciones futuras. 


\section{BIBLIOGRAFIA}

ATKINS, H. T.-Premature rupture of the membranes. A. J. O \& G. 58. 4. 1949.

CAICEDO P, H. y MUÑOZ DELGADO S.-Ruptura prematura de membranas. Leído en gran sesión de obstetricia. Clínica de Maternidad de Cali. Diciembre de 1956.

CALKINS, L. A.-Premature spontaneous rupture of the membranes. A. J. O. \& G. 64. 4. 1952.

CUADRos, A.-Anotaciones sobre inducción del trabajo de parto. Comunicación personal.

EASTMAN, N.-Obstetrics. Ed. 10. New York 1950. Appleton_century Crofts Inc.

GOLDFINE, S.-Detection of the ruptured membranes by vaginal smear. A. J. O. \& Gin. 70. 109. 1955.

GREENHILL.-Obstetrics. 1956.

GREENHILL-DE LEE-Principales and practice of Obstetrics \& Gynecology. 1947.

JAVERT CARL, T.-Bull of Margaret Hague Maternity Hospital. Aborto espontáneo y habitual, patogenia y tratamiento. Citado en la REVISTA CO. LOMBIANA DE OBSTETRICIA Y GINECOLOGIA. Número 7. Octubre y noviembre de 1956 .

LAVERGNE, J. A.-Afibrinogenemia. Primera reunión de obstetricia. Inauguración de la Maternidad del Hospital Roosevelt. Guatemala. Noviembre 21 de 1955

LULL, B. C. \& KIMBROUGH, R. A.-Obstetricia Clínica. Editorial interamericana, S. A. México. 1955.

PEREZ, M. L.-Compendio de clínica obstétrica. López \& Etchegoyen S. R. L. Buenos Aires. 1956.

ROTH, L. G.-Early rupture of membranes. A. J. O. \& G. 4. 87. 1954.

TENNENT R. A. and cols.-Surgical induction of labour in modern obs_ tetrics. The British Nedical Journal. Vol. II. 87. 1954.

VON JASCHKE, R.-Tratado de Obstetricia. Editorial Labor. 1954.

WOLTS JOHN, H. E.-A study of the usa of penicillin in premature rupture of the membranes. A. J. O. \& G. 55. 859. 1948. 